\title{
Ultrastructural observations of the incorporation of guinea-pig spermatozoa into zona-free hamster oocytes
}

\author{
C. Barros and E. Herrera \\ Embryology Laboratory, Institute of Biological Sciences, Catholic University of Chile, \\ Casilla 114-D, Santiago, Chile
}

\begin{abstract}
Summary. Zona-free hamster oocytes inseminated in vitro with acrosome-reacted guinea-pig spermatozoa were examined with the electron microscope. Guinea-pig spermatozoa, in the vicinity of the oocytes, consistently lacked the whole acrosome including the equatorial segment region. In cross fertilization sperm-egg membrane fusion does not differ significantly from that of normal fertilization. However, it was sometimes possible to observe protrusions of oocyte cytoplasm containing sperm chromatin in the process of dispersion.
\end{abstract}

\section{Introduction}

The behaviour of gamete membranes during fertilization has been studied at the ultrastructural level in the rat (Szollosi \& Ris, 1961), golden hamster (Barros \& Franklin, 1968; Yanagimachi \& Noda, 1970), rabbit (Bedford, 1972), mouse (Stefanini, Oura \& Zamboni, 1969) and man (Soupart \& Strong, 1974).

Zona-free mammalian oocytes have been observed to fuse with non-homologous spermatozoa (Hanada \& Chang, 1972; Yanagimachi, 1972; Barros, Berríos \& Herrera, 1973a, b). The behaviour of mammalian gamete membranes during cross fertilization has been only briefly reported (Barros \& Herrera, 1975). Glycerylmono-oleate-mediated fusion of a bull spermatozoon to a hen erythrocyte seems to occur as in normal fertilization, i.e. the acrosome vesiculates and the post-acrosomal area of the spermatozoon fuses with the erythrocyte (Lucy, 1975).

It seems of importance to describe in full the mechanism by which a foreign spermatozoon fuses and enters a zona-free oocyte, especially if the use of oocytes of one species (e.g. hamster) is contemplated for testing the fertilizing ability of spermatozoa of another species (e.g. man). The purpose of the present work was therefore to investigate the behaviour of the gamete membranes when zona-free hamster oocytes are inseminated in vitro with guinea-pig spermatozoa.

\section{Materials and Methods}

Adult female hamsters were induced to superovulate with 30 i.u. PMSG on the day of the postoestrous discharge (Orsini, 1961) followed by 30 i.u. HCG 3 days later. The animals were killed $16 \mathrm{~h}$ after the HCG injection and the oviducts flushed with BMOC-2 ovum culture medium (Brinster, 1971). The flushed oocytes were treated with a $0 \cdot 1 \%$ hyaluronidase solution prepared in BMOC-2, to eliminate the granulosa cells, and washed in three changes of fresh BMOC- 2 medium. Then the oocytes were treated with a $0.1 \%$ trypsin solution in BMOC-2 medium, to eliminate the zona pellucida, and washed in three changes of fresh BMOC-2.

The zona-free hamster oocytes were placed in the centre of a $50 \mu 1$ drop of BMOC-2 medium and acrosome-reacted guinea-pig spermatozoa were added (Barros, Berríos \& Herrera, 1973b). The oocytes were incubated for different times and were then fixed by the method of Karnovsky (1965) for $2 \mathrm{~h}$, post-fixed in osmium tetroxide for $45 \mathrm{~min}$, dehydrated in acetone and embedded in a lowviscosity epoxy resin (Spurr, 1969). Ultrathin sections cut on a Porter-Blum MT2B ultramicrotome were stained with uranyl acetate and lead citrate and examined with an electron microscope. 


\section{Results}

When guinea-pig spermatozoa were incubated overnight in BMOC-2 or for a few minutes between slide and coverslip the acrosomes appeared to be reacted under the phase-contrast microscope. Examination with the electron microscope showed that the spermatozoa of the two treatments did not differ in appearance. In all the spermatozoa present in the vicinity of the oocyte, the whole acrosome, i.e. the acrosomal cap and the equatorial segment, was absent. Soon after mixing acrosome-reacted guinea-pig spermatozoa with zona-free hamster oocytes, many oocyte microvilli became associated with the spermatozoon. However, only the microvilli surrounding the post-acrosomal area became fused with the spermatozoon (Pl. 1, Fig. 1). No fusion was ever observed of the many microvilli which surround the inner acrosome membrane. More advanced stages of sperm incorporation into the hamster vitellus are depicted in P1. 1, Figs 2 and 3. The fusion process progresses towards the caudal post-acrosomal region of the spermatozoon, and in the area originally occupied by the equatorial segment, the oocyte plasma membrane is more closely apposed to the inner acrosome membrane than in areas anterior to this region (P1. 1, Figs 2 and 3). The space between the inner acrosome membrane and the oocyte plasma membrane at the area originally occupied by the equatorial segment has low electron density.

Soon after fusion of the gamete membranes, the dense sperm chromatin begins to disperse from the periphery to the centre and from the middle region to the anterior and posterior ends of the sperm head. While the chromatin is in the process of dispersion, dense round bodies, $0.1-0.2 \mu \mathrm{m}$ in diameter, become visible (Pl. 1, Figs 2 and 4) but are not distinguishable later.

In many instances, observation of the zona-free hamster oocytes which had been in contact in vitro with the guinea-pig spermatozoa, under phase-contrast microscopy showed protruding vesicles at the surface of the oocyte (Pl. 2, Fig. 5). After squashing the egg it was possible to see that each protrusion was associated with a sperm tail. When such protrusions were examined with the electron microscope, it was clear that they corresponded to a vesicle with oocyte cytoplasm in whose interior it was possible to see partly dispersed sperm chromatin (Pl. 2, Fig. 6), suggesting extrusion of the oocyte cytoplasm rather than ingress of the spermatozoon. Full dispersion of the sperm chromatin was observed in the developing male pronuclei present inside the oocyte cytoplasm as well as in those protruding outside the oocyte surface (P1. 2, Fig. 7).

\section{Discussion}

From the observations reported in this work it is apparent that, in mammalian cross fertilization, gamete fusion does not significantly differ from that of normal fertilization. Gamete membrane fusion takes place caudal to the equatorial segment and involves the plasma membrane of the post-acrosomal region.

\section{EXPLANATION OF PLATE 1}

Fig. 1. The hamster oocyte microvilli have already fused with a guinea-pig spermatozoon. Fusion has occurred at the post-acrosomal area and at this point it is possible to observe many dot-like structures (arrows). The oocyte plasma membrane (em) becomes closely apposed to the inner acrosome membrane (iam) at the region originally occupied by the equatorial segment. Note the absence of the acrosome.

Figs 2 and 3. Advanced stages of sperm-egg fusion. The fusion process has advanced toward the caudal end of the post-acrosomal area. The anterior portion of the spermatozoon is still outside the egg. The process of sperm chromatin dispersion is already underway. During this process, it is possible to observe round dense bodies (db). The egg plasma membrane (em) is closely apposed to the inner acrosome membrane (iam) only at the region originally occupied by the equatorial segment. Detail of this area is shown in Fig. 3.

Fig. 4. Guinea-pig spermatozoon which has already fused with the hamster oocyte. Round and electrondense bodies $(\mathrm{db})$ are present during the process of chromatin dispersion. 

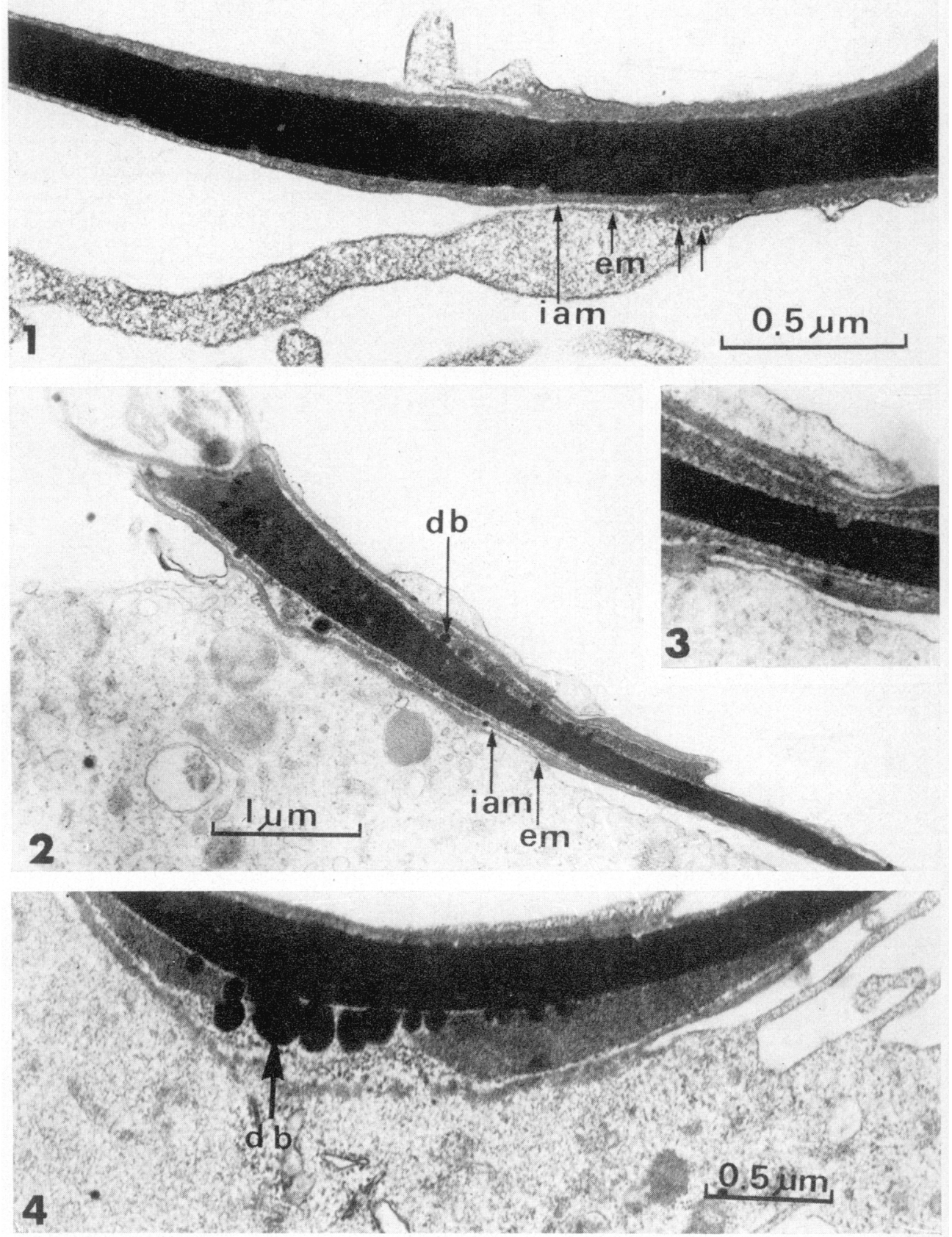
PLATE 2
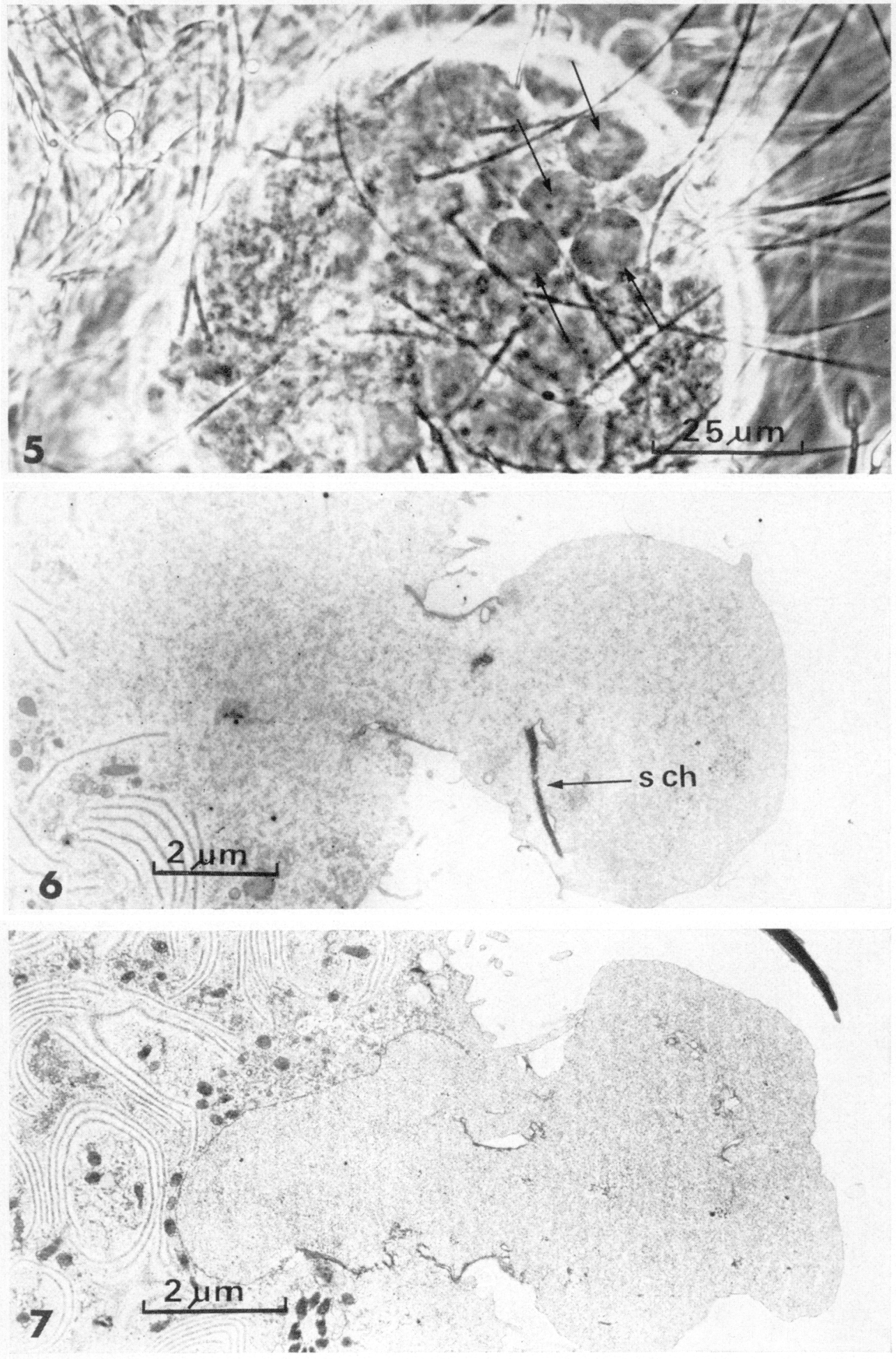
Study of the distribution of negative charge on the sperm plasma membrane shows that it is discontinuous over the surface, the tails being generally more acidic than the head regions (Yanagimachi, Noda, Fujimoto \& Nicolson, 1972). The abrupt change in the distribution of negative charge at the junction of two morphologically different segments suggests that the biochemical properties of the plasma membrane are also different in the various segments of the spermatozoon (Yanagimachi et al., 1972). The use of ferritin-conjugated agglutinins from Ricinus communis has permitted visualization of the different behaviour properties of the plasma membrane (Nicolson \& Yanagimachi, 1974) and that of the post-acrosomal area shows a higher relative mobility of lectin receptors than that of tail and acrosome. These characteristics probably confer to this region the ability to fuse not only with the egg plasma membrane but also with other kinds of cells, as in the case of a bull spermatozoon fusing with a hen erythrocyte (Lucy, 1975). However, it has to be emphasized that the ability of the post-acrosomal plasma membrane to fuse spontaneously or by induction can only be manifested after the acrosome reaction has taken place. Barros (1974) has suggested that the acrosomal enzymes might be important not only for sperm passage through the egg investments but also to allow sperm-egg membrane fusion, probably by modifying the post-acrosomal plasma membrane.

Conception of the plasma membrane as a dynamic fluid structure (Singer \& Nicolson, 1972) can explain gamete fusion. Rests of gamete membranes cannot be found at the point where both gametes are continuous. Membrane continuity is clear at the caudal end of the equatorial segment and posterior to the post-acrosomal membrane. It seems then that the sperm plasma membrane of the post-acrosomal area dissolves into the egg membrane (Yanagimachi, Nicolson, Noda \& Fujimoto, 1973).

Barros \& Franklin (1968) raised the question of the origin of the outer membrane of the desmosome-like structure of fertilizing spermatozoa present within the egg. Yanagimachi \& Noda (1970) and Barros, Fujimoto \& Yanagimachi (1973) sustained the idea that egg and sperm membrane fusion occurred at the posterior limit of the equatorial segment, after this region has vesiculated. On the other hand, Bedford (1972) believes that it is not unlikely that the equatorial segment passes intact into the egg cytoplasm.

The observations made in the present study seem to indicate that the equatorial segment does not pass intact into the egg and support the view that sperm-egg membrane fusion occurs at the posterior end of the equatorial segment rather than at the anterior end. The desmosome-like structure present in homologous fertilization might arise from a specific binding between the egg plasma membrane and the inner acrosomal membrane at the equatorial segment.

The round dense bodies that are seen among the dispersing chromatin fibres are of unknown composition and function. However, their presence does not seem to be a feature of cross fertilization since structures of similar appearance can be seen in normal homologous fertilization (see Bedford, 1972, Fig. 13, and Barros, Fujimoto \& Yanagimachi, 1973, Fig. 4).

The reaction of the golden hamster oocytes to guinea-pig spermatozoa by the formation of round large vesicles, though it is not a regular feature, occurred in many of the inseminated oocytes. No mention was made by Yanagimachi (1972) of an anomalous reaction of hamster oocytes to foreign spermatozoa, but Franklin (1965) described exaggerated fertilization cones in Arbacia punctulata oocytes inseminated with Mellita quinquiesperforata spermatozoa.

The present observations of the normality of membrane behaviour of cross-fertilizing gametes are important because zona-free mammalian oocytes are used to assess the fertilizing capacity of the spermatozoa of animals for which it is difficult to obtain homologous oocytes. This might be specially important when studying the fertilizing capacity of human spermatozoa.

\section{EXPLANATION OF PLATE 2}

Fig. 5. Phase-contrast micrograph of a zona-free hamster oocyte. Many guinea-pig spermatozoa have entered the egg cytoplasm. A few vesicles protrude outside the oocyte (arrows) and 5 are clearly visible in this photograph.

Fig. 6. One of the vesicles shown in Fig. 5, with part of the sperm chromatin (s ch) being visible within it.

Fig. 7. Guinea-pig pronucleus in which the sperm chromatin is fully dispersed. Note that the pronucleus has protruded outşidẹ the oocyte. 
This work was supported by the Ford Foundation and the Catholic University of Chile Research Fund. We also thank Dr L. Izquierdo and Dr V. Monasterio, Faculty of Sciences, University of Chile, and Dr J. Vial, Department of Cell Biology, Catholic University of Chile, for allowing us to use the electron microscope facilities.

\section{References}

BARros, C. (1974) Capacitation of mammalian spermatozoa. In Physiology and Genetics of Reproduction, part B, pp. 3-24. Eds E. M. Coutinho \& F. Fuchs. Plenum Press, New York.

Barros, C. \& Franklin, L.E. (1968) Behavior of the gamete membranes during sperm entry into the mammalian egg. $J$. Cell Biol. 37, C13-C18.

Barros, C. \& Herrera, E. (1975) Conducta de las membranas gaméticas en la fecundación cruzada. Proc. 18th A. Meeting Soc. Biol. Chile, Santiago. Abstr. No. R6.

Barros, C., Berrfos, M. \& Herrera, E. (1973a) Capacitación en mamíferos con especial referencia al espermio de cobayo. Archos Biol. Med. exp. 9, R4, Abstr.

Barros, C., Berríos, M. \& Herrera, E. (1973b) Capacitation in vitro of guinea-pig spermatozoa in a saline solution. J. Reprod. Fert. 34, 547-549.

Barros, C., Fujimoto, M. \& Yanagimachi, R. (1973) Failure of zona penetration of hamster spermatozoa after prolonged preincubation in a blood serum fraction. $J$. Reprod. Fert. 35, 89-95.

BEDFORD, J.M. (1972) An electron microscopic study of sperm penetration into the rabbit egg after natural mating. Am. J. Anat. 133, 213-254.

Brinster, R.L. (1971) Mammalian embryo metabolism. In The Biology of the Blastocyst, pp. 303-318. Ed. R. J. Blandau. University of Chicago Press.

Franklin, L.E. (1965) Morphology of gamete membrane fusion and of sperm entry into oocytes of the sea-urchin. J. Cell Biol. 25, 81-100.

Hanada, A. \& Chang, M.C. (1972) Penetration of zona-free eggs by spermatozoa of different species. Biol. Reprod. 6, 300-309.

KARNOVSKY, M.J. (1965) A formaldehyde-glutaraldehyde fixative of high osmolality for use in electron microscopy. J. Cell Biol. 27, 137A-138A.

LUCY, J.A. (1975) Aspects of the fusion of cells in vitro without viruses. J. Reprod. Fert. 44, 193-205.
Nicolson, G.L. \& Yanagimachi, R. (1974) Mobility and the restriction of mobility of plasma membrane lectin-binding components. Science, N.Y. 184, 1294-1296.

ORsinI, M.W. (1961) The external vaginal phenomena characterizing the stages of the estrous cycle, pregnancy, pseudopregnancy, lactation and the anestrous hamster, Mesocricetus auratus, Waterhouse. Proc. anim. Care Panel 11, 193-206.

Singer, S.J. \& Nicolson, G.L. (1972) The fluid mosaic model of the structure of cell membranes. Science, N.Y. 175, 720-731.

Soupart, P. \& Strong, P.A. (1974) Ultrastructural observations on human oocytes fertilized in vitro. Fert. Steril. 25, 11-43.

SpURR, A.R. (1969) A low-viscosity epoxy resin embedding medium for electron microscopy. J. Ultrastruct. Res. 26, 31-43.

Stefanini, M., OURA, C. \& Zamboni, L. (1969) Ultrastructure of fertilization in the mouse. 2. Penetration of sperm into the ovum. J. submicrosc. Cytol. 1, 1-23.

Szollosi, D.G. \& Ris, H. (1961) Observations on sperm penetration in the rat. J. biophys. biochem. Cytol. 10, 275-283.

YANAGIMACHI, R. (1972) Penetration of guinea-pig spermatozoa into hamster eggs in vitro. J. Reprod. Fert. 28, 477-480.

YANAGIMACH, R. \& NodA, Y.D. (1970) Electron microscope studies of sperm incorporation into the golden hamster egg. Am. J. Anat. 128, 429-462.

YANAGIMACHI, R., NodA, Y.D., Fujimoto, M. \& NicolsoN, G.L. (1972) The distribution of negative surface charges on mammalian spermatozoa. Am. $J$. Anat. 135, 497-520.

YaNAGIMACH, R., Nicolson, G.L., NodA, Y.D. \& Fujimoto, M. (1973) Electron microscopic observations of the distribution of acidic anionic residues on hamster spermatozoa and eggs before and during fertilization. J. Ultrastruct. Res. 34, 344-353.

Received 18 May 1976 